\title{
Synthesis and design of a one-degree-of-freedom planar deployable mechanism with a large expansion ratio
}

\author{
David St-Onge* \\ Département de génie mécanique \\ Université Laval \\ Québec, Québec, Canada \\ Email: david.st-onge.2@ulaval.ca \\ Clément Gosselin \\ Département de génie mécanique \\ Université Laval \\ Québec, Québec, Canada \\ Email: gosselin@gmc.ulaval.ca
}

This paper presents a new design of a deployable onedegree-of-freedom mechanism. Polygonal rigid-link designs are first investigated. Then, belt-driven links are considered in order to maximize the expansion ratio while avoiding flattened ill-conditioned parallelogram configurations. The planar base shape of the proposed design is a triangle. Hence, virtually any planar or spatial surface can be created by assembling such faces. For architecture and telescopic applications the cupola assembly is investigated. The advantages of this approach are discussed and the scalability is demonstrated. Finally, a prototype is built for illustration purposes.

\section{Introduction}

Deployable mechanisms have a wide range of applications, namely transformable architectures $[1,2]$ and space applications, where the dimensions at launch are limited [3,4]. Amongst the design criteria of such mechanisms are: the structural stability of the deployed shape, the robustness of the structure while it is deployed, the complexity of its actuation and of course the geometry.

Many design solutions were proposed to fulfill the latter, e.g., the Hoberman designs, which expand a star to a sphere [5], designs based on 8-bar mechanisms [6] or even umbrella-like designs $[7,8]$. To achieve greater expansion ratios, some authors proposed linear deployment using a telescopic mast or some specific cable-support structure design [3]. However, the geometry of all of the above mentioned mechanisms changes during their expansion. To avoid unstable configurations during the deployment a solution is to preserve the overall shape all along. Polyhedral linkages aim to provide such property.

For instance, overlapping scissors [9], 6-bar scissors [10] or a mechanism replacing each edge of a polyhedron by a prismatic link [11] are such mechanisms. Another approach, inspired by virus geometry, consists in deploying a mechanism with planar links between rotating faces [12] or for some simple models, only translating faces [13].

In [14], one-degree-of-freedom expanding polyhedra with articulated faces were developed. Several Platonic polyhedra were designed and some prototypes were built, amongst which the icosahedron, a good approximation of a sphere with triangular faces as shown in Fig.1.

The aim of the current research is to reach a high expansion ratio while preserving the geometry. As seen in Tab.1, the performances of such mechanisms are very limited in terms of expansion ratio. For instance, the articulated triangles developed in [14] are limited by a maximum theoretical ratio of 4.46 , but the prototypes built never reached a ratio above 3.26 .

\begin{tabular}{ccc}
\hline Model & Ratio & Shape preserved \\
\hline Hoberman sphere & 3.2 & $\mathrm{~N}$ \\
Wohlhart Polyhedral Link [15] & $<2$ & $\mathrm{Y}$ \\
Wei parallelogram [9] & $<1.5$ & $\mathrm{Y}$ \\
1DoF planar triangle [14] & 3.26 & $\mathrm{Y}$ \\
\hline
\end{tabular}

Table 1. Expansion ratio of some concept mechanims

In this paper, deployable planar mechanisms with large

*Address all correspondence to this author. 

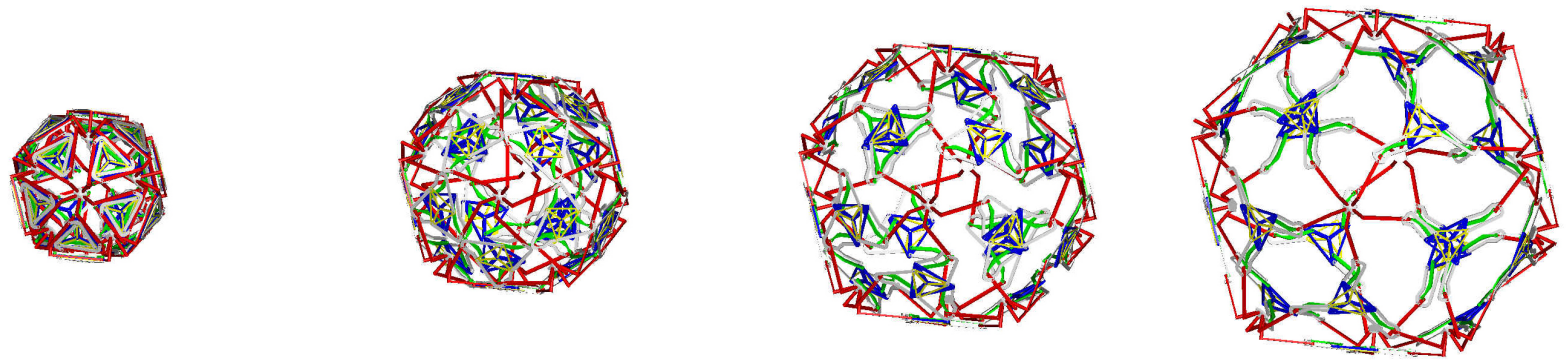

Fig. 1. Deployment of an icosahedron with articulated faces (from [14])

expansion ratios are developed. First, the concept of mobility and overconstraint is briefly reviewed. Then, an architecture similar to that found in [14] is proposed in order to reach large expansion ratios and the kinematic equations are developed in order to study the transmission ratio throughout deployment. The use of belt transmissions is then introduced to provide a convenient practical implementation. The optimization of the mechanism is then addressed through an investigation of the impact of the different parameters. A mechanism is also proposed in order to assemble several mechanism units into a foldable system. Finally, a prototype is described that illustrates the viability of the proposed concept.

\section{Mobility}

When designing a mechanism, it is useful to compute the degree of mobility or overconstraint (if smaller than zero) $l$ of the mechanism. The latter is obtained using the Chebychev-Grübler-Kutzbach formula, namely [16]:

$$
l=d(m-g-1)+\sum_{i=1}^{g} e_{i}
$$

where $d$ is the number of degres-of-freedom (DoF) of an unconstrained rigid body ( 6 for a spatial mechanism), $m$ is the number of rigid bodies, $g$ the number of joints, and $e_{i}$ is the number of degree of freedom of the ith joint [14]. In this work only revolute joints, each having a single degre of freedom are used, and thus Eqn. (1) simplifies to:

$$
l=6(m-1)-5 g
$$

All the mechanisms studied in this paper are overconstrained, i.e, they lead to a negative mobility when Eqn. (2) is applied. The latter are reported in Tab.2.

Although it is well known that the Chebychev-GrüblerKutzbach mobility criterion cannot predict the actual mobility of overconstrained mechanisms $[17,18]$, this criterion is useful to assess the degree of overconstraint. Some authors

\begin{tabular}{cccccc}
\hline Mechanisml & faces & $m$ & $g$ & $l$ & $R_{M}$ \\
\hline Original rigid-link (closed) & 20 & 292 & 440 & -454 & 3.26 \\
Original rigid-link (open) & 5 & 76 & 110 & -100 & 3.26 \\
Enhanced rigid-link (open) & 5 & 136 & 200 & -190 & 5 \\
Belt-driven (open) & 5 & 166 & 245 & -235 & 5.6 \\
\hline
\end{tabular}

Table 2. Mobility of the mechanisms studied in this paper according to the Chebychev-Grübler-Kutzbach formula

also argue that such criteria are far from acceptable for multiloop mechanisms [19]. The Chebychev-Grübler-Kutzbach mobility is presented here as a comparison between designs instead of an absolute measurement of mobility or constraint. The first line of Tab. 2 corresponds to the mechanism of Fig.1 while the second corresponds to a mechanism using only five planar triangular faces to form an open cupola. As expected the open geometry leads to a degree of overconstraint smaller than that of the icosahedron. The last two lines of Tab. 2 will be referred to in upcoming sections of the paper.

\section{Increasing the expansion ratio of planar modules}

Fig.2 presents the details of a triangular face of the mechanism described in [14]. The bodies (1), (4), (5) and (6) form a four-bar mechanism often designed to be a parallelogram. In order to increase the expansion ratio of this planar mechanism, the parallelogram linkages can be replicated, as shown in Fig.3.

Theoretical and simulated expansion ratios larger than 5 are obtained by adding only two stages (or parallelograms). Stages increase the degree of overconstraint (Tab.2 - line 3) and friction but the resulting mechanism still has a single degree of freedom. The design of the stages proposed in [14] was revised to improve compactness as shown in Fig.4 and to ensure that fewer stages are required to reach the same large expansion ratio.

The leg mechanism shown in Fig.3 can theoretically reach an expansion ratio of 7.92 [14], assuming that all links of a leg can be superimposed in its retracted position. However, manufacturing contraints and mechanical interferences 


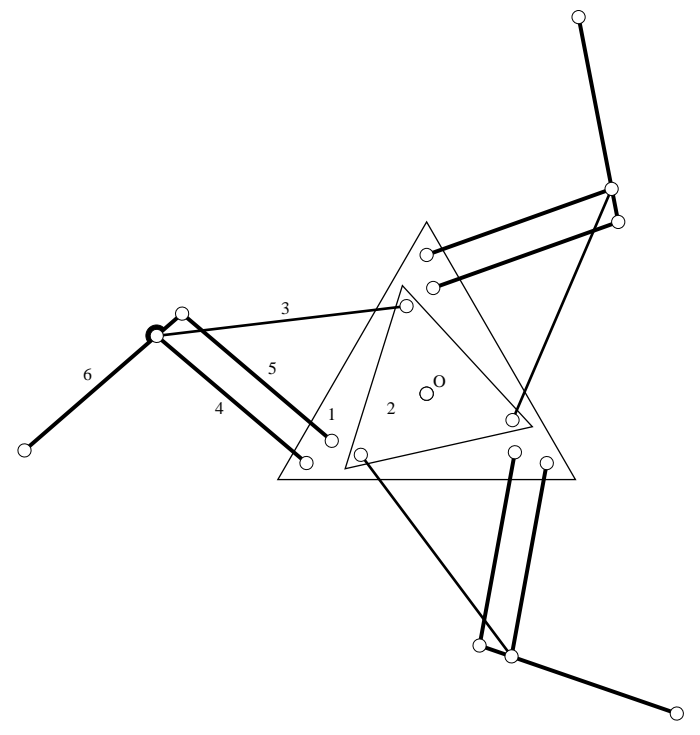

Fig. 2. Schematic representation of a triangular face from [14].

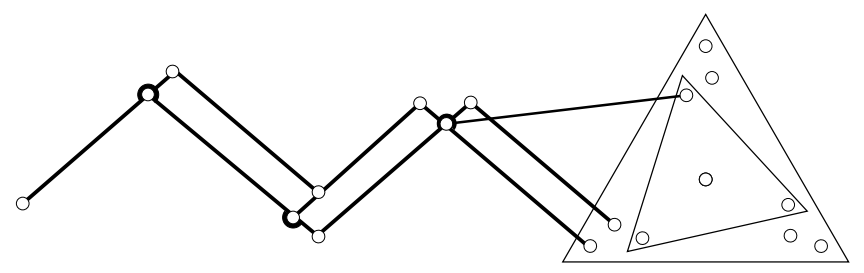

Fig. 3. Schematic representation of a deployable leg with large expansion ratio and compact design.

limit the ratio to approximately 5 , see $R_{M}$ in Tab.2. For instance, the prototype design shown in Fig.4 was constrained by its manufacturability with a conventional $3 \mathrm{D}$ printer and by the use of small but widely available steel shoulder screws for its joints (\#4-40, 1/4" long) to create a smooth interface (steel-ABS instead of ABS-ABS).

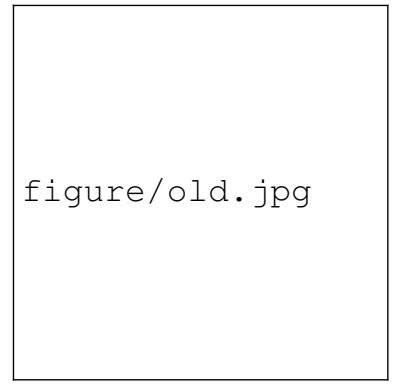

(a)

Fig. 4. Retracted triangular 1-dof deployable mechanism : (a) 1 stage per leg from [14] (b) 3 stages per leg optimised.

The prototype provides an excellent functionality of the joints and sufficient structural strength of the links. The simulation shows no singular positions and a proper torque (velocity) transmission ratio (Fig.5). However, the completely retracted position appears to have a poor force transmission on the prototype. With a large enough force applied to the actuator while retracting, the leg could, by bending imperceptibly its links, reach a retracted position so close to the geometric singularity that the mechanism would block. Then inducing manually vibrations would allow it to jump over that position. Hence, in order to further examine the mechanical properties of the mechanism an acceptable range of the transmission angles is prescribed.

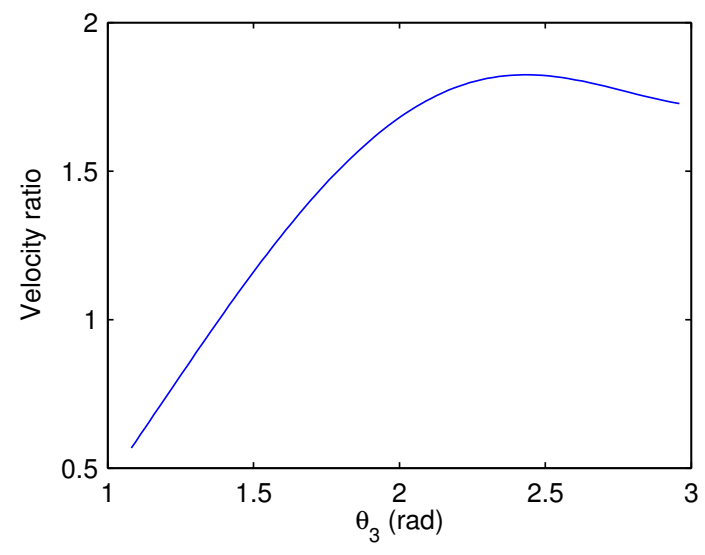

Fig. 5. Velocity transmission ratio for all deployment positions.

The transmission angle is defined as the angle between the direction of the driving velocity and the direction of the output velocity of a mechanism [20]. The impact of the transmission angle and the range of its acceptable values are still the subject of research [21,22]. A generally acceptable range of $\pi / 2 \pm \pi / 4$ specific to the geometric definition of the angles is used here. The leg of the deployable mechanisms can be divided in two stages, namely the actuation stage, which consists of a five-bar mechanism and then a series of four-bar mechanisms. The actuation stage and a first four-bar module are illustrated schematically in Fig.6. Balli and Chand [21] indicate how the transmission angle should be measured in four-bar and five-bar planar linkages. Based on their definition, angles $\mu_{A}$ and $\mu_{P}$, shown in Fig.6 are used here as transmission angles.

The first stage is represented in its general form in Fig.7. Here, its transmission angle is $\mu_{A}=\pi-\theta_{3}$. Since the value of the input angle $\theta_{1}$ is known along the deployment trajectory, the problem amounts to finding the pose of bar $E B C$ for given positions of points $A, F$ and $D$, with respect to a fixed frame defined at A. The latter is obtained through a rotation $(\boldsymbol{Q})$ and a translation $(\boldsymbol{d})$ of the fixed frame at point $O$, namely

$$
\begin{aligned}
\boldsymbol{p}_{x_{1}, y_{1}} & =\boldsymbol{Q}^{T}\left(\boldsymbol{p}_{x, y}-\boldsymbol{d}\right) \\
\boldsymbol{Q} & =\left[\begin{array}{cc}
\cos \theta_{1}-\sin \theta_{1} \\
\sin \theta_{1} & \cos \theta_{1}
\end{array}\right] \\
\boldsymbol{d} & =\left[\begin{array}{l}
l_{1} \cos \theta_{1} \\
l_{1} \sin \theta_{1}
\end{array}\right],
\end{aligned}
$$




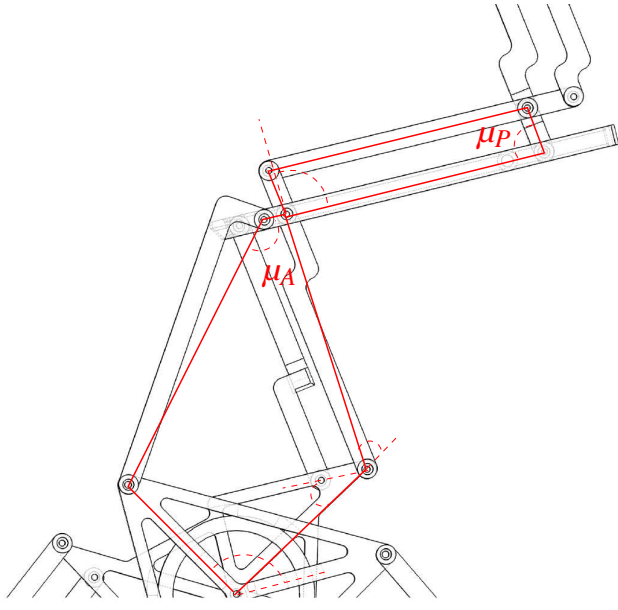

Fig. 6. Transmission angles and kinematic loops. The parallelogram included in the actuation module is not highlighted.

where $\boldsymbol{p}_{x, y}$ is the posistion vector of a point on bar EBC in the fixed frame at $O$ and $\boldsymbol{p}_{x_{1}, y_{1}}$ is theposition vector of the same point expressed in the frame $A_{x_{1}, y_{1}}$ with origin at $A$. The solution of the above problem is found using the approach described in [23]. To this end, the coordinates of points $B, D$ and $F$ are first written respectively as $B_{x}, B_{y} \ldots .$. and $F_{y}$ in the reference frame $x_{1} y_{1}$. The constraints corresponding to links $A B, C D$ and $E F$ can then be written as

$$
\begin{aligned}
& l_{2}^{2}=B_{x}^{2}+B_{y}^{2} \\
& l_{4}^{2}=\left(B_{x}+l_{3} \cos \phi-D_{x}\right)^{2}+\left(B_{y}+l_{3} \sin \phi-D_{y}\right)^{2} \\
& l_{6}^{2}=\left(B_{x}-l_{7} \cos \phi-F_{x}\right)^{2}+\left(B_{y}-l_{7} \sin \phi-F_{y}\right)^{2}
\end{aligned}
$$

where $\phi$ is the angle defined between axis $x_{1}$ and link $E B C$, as shown in Fig. 7 and where $l_{2}, l_{3}, l_{4}, l_{6}$ and $l_{7}$ are defined in Fig. 7. According to the procedure described in [23], Eqn. 6 is then subtracted from Eqns. 7 and 8 and the two equations thereby obtained are readily solved for $B_{x}$ and $B_{y}$, leading to

$$
\begin{gathered}
B_{x}=\frac{R_{3} R_{5}-R_{6} R_{2}}{R_{4} R_{2}-R_{5} R_{1}} \\
B_{y}=\frac{R_{6} R_{1}-R_{4} R_{3}}{R_{4} R_{2}-R_{5} R_{1}}
\end{gathered}
$$

with

$$
\begin{gathered}
R_{1}=2 l_{3} c_{\phi}-2 D_{x} \\
R_{2}=2 l_{3} s_{\phi}-2 D_{y} \\
R_{3}=l_{3}^{2}+D_{x}^{2}+D_{y}^{2}-2 D_{x} l_{3} c_{\phi}-2 D_{y} l_{3} s_{\phi}-l_{4}^{2}+l_{2}^{2} \\
R_{4}=-2 l_{7} c_{\phi}-2 F_{x} \\
R_{5}=-2 l_{7} s_{\phi}-2 F_{y} \\
R_{6}=l_{7}^{2}+F_{x}^{2}+F_{y}^{2}+2 F_{x} l_{7} c_{\phi}+2 F_{y} l_{7} s_{\phi}-l_{6}^{2}+l_{2}^{2}
\end{gathered}
$$

where $s_{\phi}$ and $c_{\phi}$ respectively stand for the sine and cosine of angle $\phi$. Eqns. 9 and 10 are then substituted into Eqn. 6 , which leads to an expression that contains only one unknown, namely angle $\phi$. Using the tangent of the half angle substitution, the latter expression can be converted into a polynomial of degree 6 , as shown in [23]. As shown in the latter reference, the solution of this polynomial can yield up to 6 real roots. The detailed expressions of the coefficients of the polynomial are not given here due to space limitation.

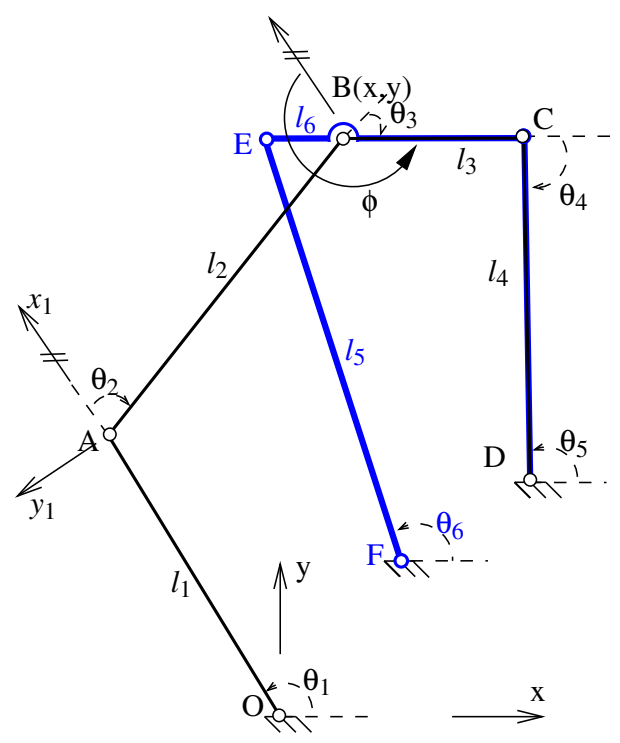

Fig. 7. Actuation stage.

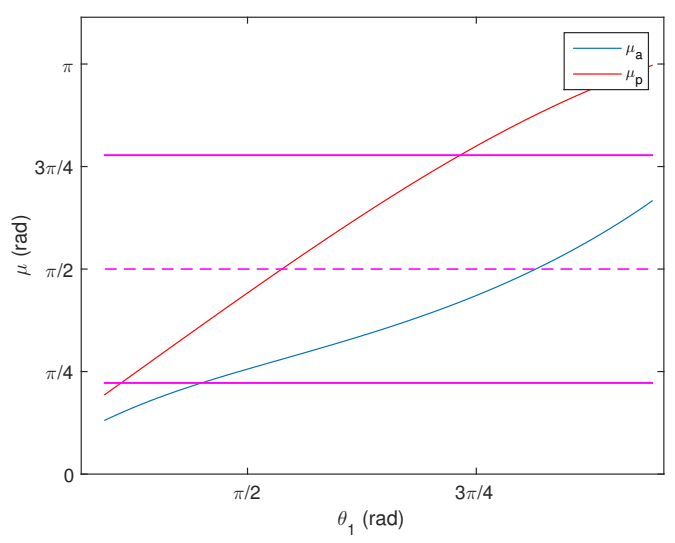

Fig. 8. Evolution of the transmission angles through deployment.

Simulating the full deployment of a leg, Fig.8 presents the resulting transmission angles. It can be observed that the transmission ratio goes beyond the acceptable limits (represented by the horizontal lines). This is confirmed experimentally, i.e., the mechanism does not deploy smoothly. The transmission angle of the actuation stage goes above the up- 
per limit when fully retracted $\left(\theta_{1}\right.$ close to $\left.\pi\right)$ as shown in Fig.8.

Keeping the transmission angles within the acceptable limits while achieving an expansion ratio above 5 was found unrealistic with this design. A new transmission paradigm eliminating the need for rigid parallelograms is required to fulfill these requirements.

\section{Belt-driven mechanisms}

It is well known (see for instance [24]), that linkage mechanisms have equivalent belt-driven counterparts. Taken separately, each leg of the triangle can be viewed as a serial kinematic chain and thus passing from a linkage-driven mechanism to a tendon-pulley architecture is straightforward.

The rigid link model developed above (four-bar and five-bar mechanisms) can be converted almost directly to a pulley-belt mechanism, as shown in Fig.9. The pulleys and the respective joints share the same axis, yet, they can freely rotate around it without transmitting the rotation to the link. Each member has a fixed pulley on each end, and the driving belts connect two non adjacent members as shown in Fig.10. For instance, member 1 drives member 3 , while member 2 is driven by the base, thus the mechanism has only one degree of freedom. The equivalence appears in the Jacobian, which is the same for a four-bar stage and a belt-driven link.

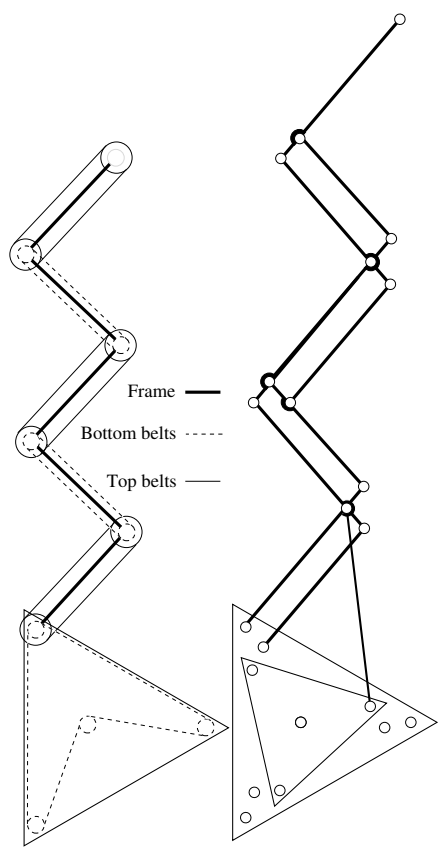

Fig. 9. Equivalence between the belt-driven and the rigid-link mechanisms.

Compared to the previous enhanced version of a rigidlink mechanism (Fig.3), the belt-driven mechanism of Fig.9 has one more stage on each leg, which explains its lower mobility shown as shown in Tab.2. However, in pratice, the two designs are equivalent.
Since the belts are flexible, some extension may occur, which is considered negligible. A constant tension, which depends on the length and material of the links, is maintained. In such a design the belts are used for movement and force transmission over a small range of motion. Thus, one criterion for selecting the belt is the ratio of its tooth width to the motion range. A full deployment of the mechanism corresponds to a rotation of each pulley of $\pi$ radians relative to their specific reference, which for the prototype represents a displacement of less than $4 \%^{1}$ of each belt length. As discussed for the previous mechanism, the transmission ratio of the forces throught the legs has a major impact on the functionnality of the design. For the belt-driven stages, the product of pulley ratios is the only relevant parameter. If each stage as a ratio $\frac{R_{\text {out }}}{R_{\text {in }}}<1$, the central actuator will need to perform more work to counter friction in the joints, but a ratio greater than one would help. For pratical reasons only one size of pulley is used in the prototype and therefore the ratio of the pulley diameters is equal to one. This also simplifies the deployment design since all stages have the same deployment rate.

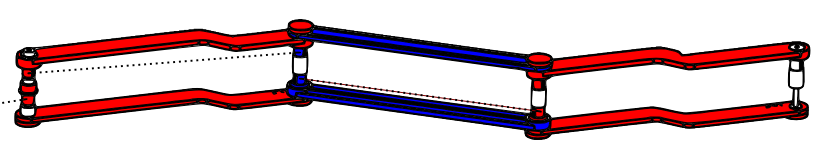

Fig. 10. Belt-driven mechanism (belts are dot lines).

\section{Geometric analysis and effect of the parameters}

Before a numerical optimisation is undertaken, the effect of the different geometric parameters is assessed, in order to guide the formulation of the problem. First, the concept of virtual triangle is introduced. The latter is defined as the triangle whose vertices are the end point of each of the deploying legs of the triangular mechanism, as illustrated in Fig. 11. If multiple triangular mechanisms are assembled to form a planar surface, then all links must remain within the virtual triangle throughout the deployment in order to avoid mechanical interferences between neighbouring triangular mechanisms.

However, in most assemblies the triangular faces are not in the same plane. It is then actually possible for the links to extend beyond the virtual triangle on one half of its edges without mechanically interfering (Fig.11).

The effect of each of the geometric parameters on the expansion ratio is now studied. The expansion ratio is defined as the ratio of the height of the triangular mechanism in its fully deployed configuration to the height of the triangular mechanism in its retracted configuration. The retracted

\footnotetext{
${ }^{1}$ mean belt length of $373 \mathrm{~mm}$ and pulley diameter of $8.6 \mathrm{~mm}$
} 


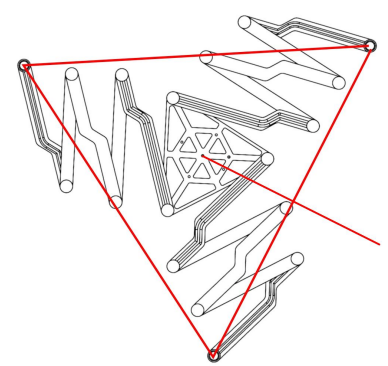

(a)

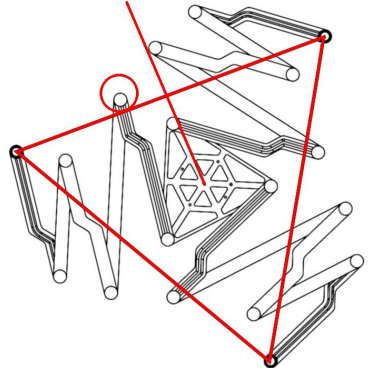

(b)
Fig. 11. Virtual triangle limits: (a) correct, (b) incorrect (assuming a spatial arrangement of neighbouring triangles).

height, noted $H_{R}$ can be computed using

$H_{R}=h_{T}+\sqrt{\left[\frac{w}{2}(n+1)\right]^{2}+\left[-\sum_{i=1}^{n}(-1)^{i} l_{i}-2 h_{T} \cos (\pi / 6)\right]^{2}}$,

where $h_{T}$ is the heigth of the fixed inner triangle, $w$ the width of the links, $l_{i}$ is the height of the $i$ th link and $n$ the number of links. Similarly, the height of the triangular mechanism in its fully deployed configuration is computed using

$$
H_{D}^{2}=h_{T}^{2}+\left(\sum_{i=1}^{n} l_{i}\right)^{2}-2 h_{T} \cos (5 \pi / 6) \sum_{i=1}^{n} l_{i}
$$

From inspection and iterative trials, an approximate and not necessarily optimal relation can be developed between the link lengths. The latter was empirically found to be close to

$$
\sum_{i=1}^{n} l_{i} \approx n l_{1}+(n-1) w
$$

and

$$
-\sum_{i=1}^{n}(-1)^{i} l_{i} \approx l_{1}+(n-5) \frac{w}{2}+w
$$

The planar expansion ratio to be maximized is then written as

$$
R_{P}=\frac{H_{D}}{H_{R}}
$$

which, from the above equations, only depends on the number of stages, the link thickness, the height of the inner triangle and the link lengths.

\subsection{Number of stages $(n)$}

As discussed earlier for the rigid-link architecture, the number of stages in each leg directly affects the expansion

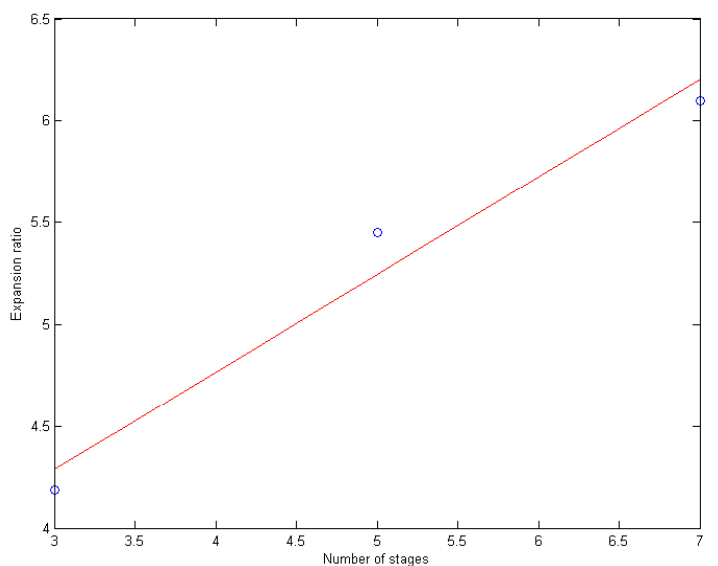

Fig. 12. Expansion ratio as a function of the number of stages for $h_{T}=90 \mathrm{~mm}$ and $w=17 \mathrm{~mm}$.

ratio. Since a larger number of stages also increases the number of joints and hence the complexity of the mechanism, a reasonable maximum number of stages should be set depending on the context. For instance, for a fixed inner triangle height of $90 \mathrm{~mm}$ and a link thickness of $17 \mathrm{~mm}$, an increase of approximately 0.5 of the expansion ratio is observed for each new stage. This is represented in Fig.12, where the expansion ratio is plotted against the number of stages, for the above mentioned parameters. It can be noted that the expansion ratio increases linearly with the number of stages, as expected.

\subsection{Thickness of the links $(w)$}

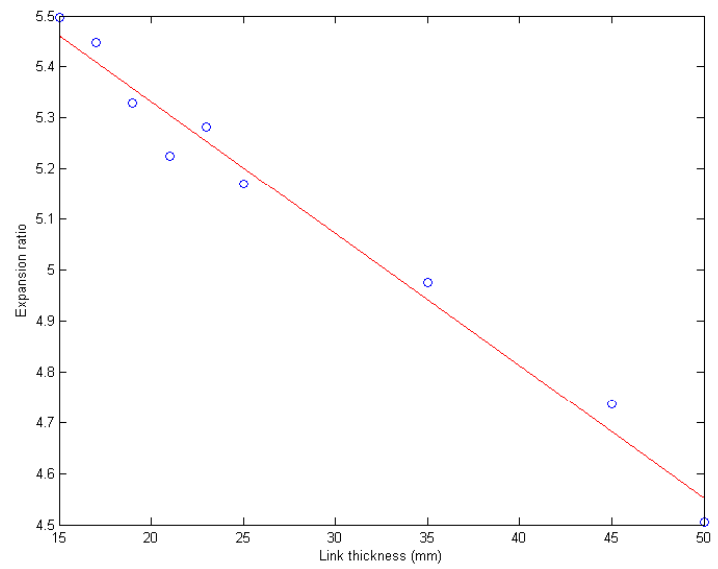

Fig. 13. Expansion ratio as a function of the link stiffness for $n=5$ and $h_{t}=90 \mathrm{~mm}$.

The thickness of the links also has an effect on the expansion ratio. While it does not make a difference on the fully extended mechanism's length, it does for its retracted position. For a fixed inner triangle height of $90 \mathrm{~mm}$ and a fixed number of links of 5 , a reduction of 0.001 of the expansion ratio is observed for an increase of $10 \mathrm{~mm}$ in link 
thickness. This is illustrated in Fig. 13, where the expansion ratio is plotted against the thickness of the links. Thus this parameter is not the most important. It is therefore chosen as the minimum value that ensures enough structural resistance for a given load and a set of pulleys. Instead of using the expansion ratio of Eqn. 15, the difference between the extended length and the retracted one $(\delta L)$ simplifies and can be differentiated with respect to the link thickness in order to get insights on the effect of this parameter. After simplification, one obtains:

$$
\frac{\partial \delta L}{\partial w} \approx \frac{(n+1)}{2}
$$

which shows that the impact of $w$ decreases with the growing number of links $n$.

\subsection{Height of the inner triangle $\left(h_{t}\right)$}

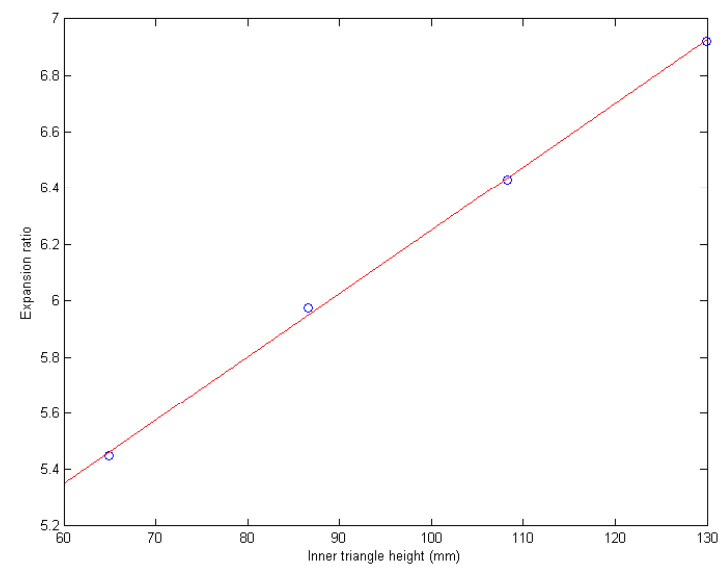

Fig. 14. Expansion ratio as a function of the height of the inner triangle for $w=17 \mathrm{~mm}$ and $n=5$.

The height of the inner triangle appears in the expression of both the retracted and the expanded heights. Therefore, its apparent direct impact on to the expansion ratio may seem limited. However, changing this parameter requires to adapt all link lengths and thus it is in fact the parameter with the greatest influence. For a fixed number of links of 5 and a fixed link thickness of $17 \mathrm{~mm}$, the expansion ratio increases by 0.002 for each $10 \mathrm{~mm}$ increase of the triangle height. This is represented in Fig. 14 where the expansion ratio is plotted as a function of the inner triangle height. The order of magnitude of the influence of the height of the inner triangle to the expansion ratio may seem comparable to the influence of the thickness of the links from the figures, nevertheless it is easier to change the inner triangle height in a wide range of values than to change the link thickness. Indeed, decreasing the latter directly impacts the robustness of the mechanism.

\subsection{Mechanism optimisation}

Based on the observations of the preceding section, an optimisation problem can be formulated. As mentioned above, the influence of $w$ is weak and hence this parameter is fixed to a reasonable value that ensures the robustness of the links. Then, it is assumed that the retracted height of the mechanism is prescribed. Eqn.11 can then be used to solve for the inner triangle height, $h_{t}$ which is then expressed as a function of $n$ and of each of the link lengths. Now from Eqns.13 and 14 we can estimate the sum of the link lengths as a function of the first length and the number of links. Substituting this result in the expression obtained for $h_{t}$ and in Eqn.12, the optimisation can be formulated as maximising $H_{D}$ over $n$ and $l_{1}$. Due to the simplications

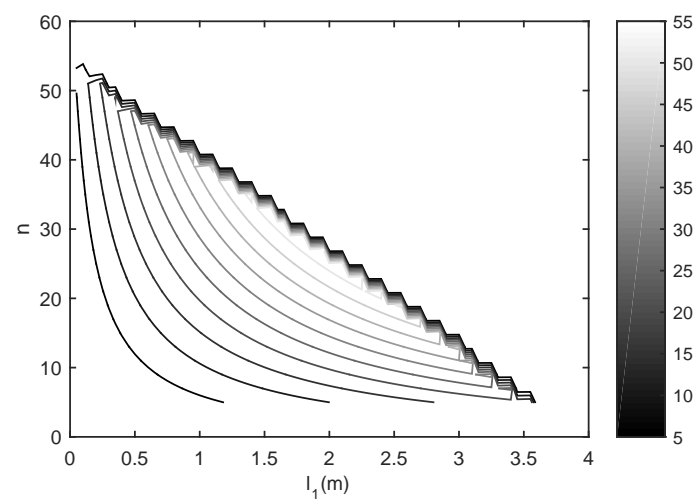

Fig. 15. Evolution of the expansion ratio according to the number of links $n$ and the length of the first link $l_{1}$ with a link width of $60 \mathrm{~mm}$ to fit when retracted a cylinder of $4.5 \mathrm{~m}$ diameter.

and approximations made upstream from the optimisation, the resulting design should be carefuly simulated to ensure geometric coherence. As shown in Fig.15, the optimisation problem described above was applied for a space application in which the deployable mechanism has to initially fit in a shuttle of $4.5 \mathrm{~m}$ diameter [25]. The upper right triangle of the plot is a domain where the retracted triangle cannot fit the given space. The result of the optimisation is an inner triangle height of $2.1 \mathrm{~m}$ with 25 links of $60 \mathrm{~mm}$ thickness. The expansion ratio obtained is above 20 .

\section{Surface assembly}

Several triangular deployable mechanisms can be connected to form more complex surfaces. The effective expansion ratio should consider the global surface. Theoretically, any planar surface can be produced as long as a triangular unit can tessellate it. When the mechanisms are assembled in a plane, the global expansion ratio will still be the same as that of the individual units. An example with 6 coplanar triangular mechanisms is shown in Fig.16. The main issue with such an assembly is its lack of ressitance to forces perpendicular to the plane when fully extended. Also, as mentioned above, all links must remain within the virtual triangle 
throughout the deployment. For earth applications the links would have to be very light and very stiff to ensure robustness. In space, the weight of the links is not an issue and hence it could be suitable to extend a sail for instance.

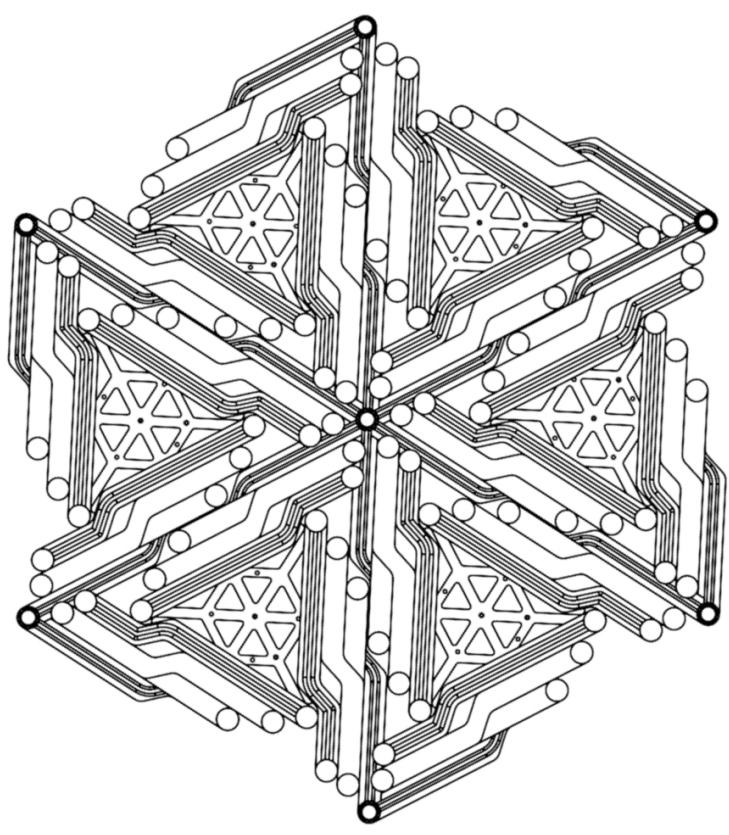

Fig. 16. Planar assembly.

For architectural applications (roof, shelters) and some space applications (aerobraking, debris net, telescope), the most suitable shape is a cupola as shown in Fig.17. Compared to a flat design, the cupola has the advantage of being more structurally robust to surface buckling. Previous work succesfully demonstrated the use of closed-loop cables or belts to deploy panels in space [26]. However, all of the previously mentioned works studied closed geometries and one may argue that an open configuration would be less stable. Wohlhart studied the cupola configuration for some of his mechanisms $[15,27]$. He observed from using open geometries with various mechanisms that they can be unstable, but that mirroring the mechanism on each face makes the system stable enough.

Assembling an icosahedral shape to approximate a cupola leads to a different expansion ratio for the 3D shape. The solid expansion ratio of the cupola is set to be the ratio of its extended to its retracted radius. The expansion ratio $R$ is computed from the radius of an inscribed sphere, namely

$$
\begin{gathered}
r_{S i}=\frac{\alpha^{2} a_{i}}{2 \sqrt{3}}+\frac{e}{2} \\
R_{C}=\frac{r_{S D}}{r_{S R}}=\alpha^{2}\left[\frac{R_{P}+\frac{\sqrt{3}}{\alpha^{2}} \frac{e}{a_{R}}}{1+\frac{\sqrt{3}}{\alpha^{2}} \frac{e}{a_{R}}}\right]
\end{gathered}
$$

where $\alpha$ is the golden ratio $\left(\frac{1+\sqrt{5}}{2}\right), a_{R}$ the side of a triangular face when retracted and $e$ the thickness of a face. Except for

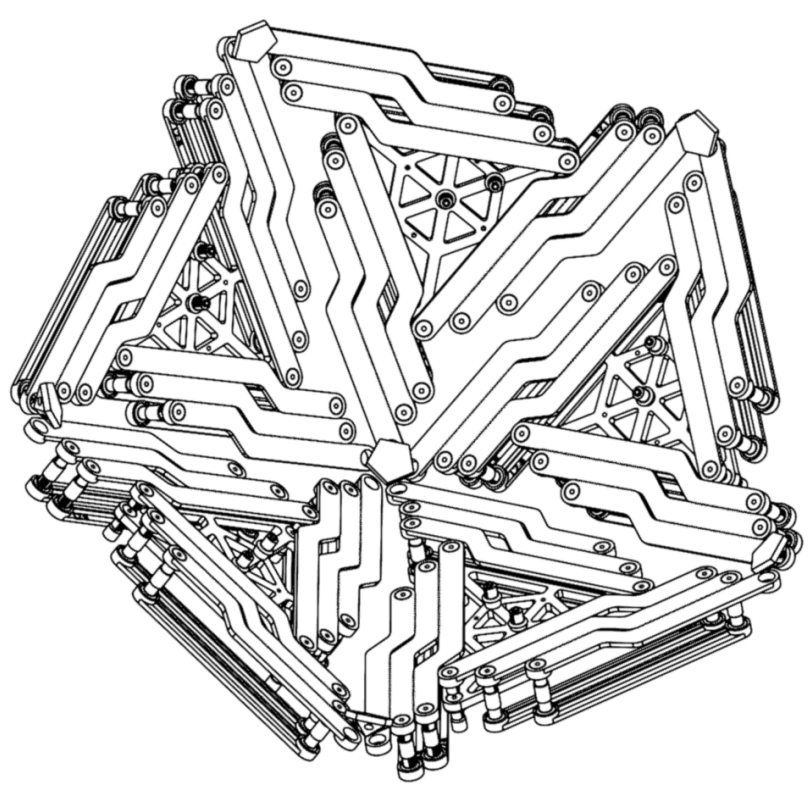

Fig. 17. Cupola assembly.

some constant values, the difference between the planar expansion ratio $R_{P}$ given in Eqn.15 and the above cupola ratio $R_{C}$ resides in the effect of the mechanism thickness (perpendicular to the extension plane). If the ratio $\frac{e}{a_{R}}$ increases the cupola expansion ratio decreases.

\subsection{Dual phase rotary joint}

Unfortunately, the cupola assembly leads to a global expansion ratio smaller than the expansion ratio of its faces. However, there is a way to take advantage of repetitive geometry (pattern) of the design. Indeed, all the identical triangular faces can be piled to fit in a smaller volume for transit. This approach requires a system to unfold each face before it can be deployed. The main challenge with this deployment strategy is to design a joint that allows a $180^{\circ}$ rotation around an edge (Fig.18-a) and then locks to a new rotation axis to allow the joint rotation perpendicular to the face plane (Fig.18-d).

The mechanism proposed in Fig. 18 makes use of loaded springs to unfold and to retract parts blocking the second rotation axis and of a permanent magnet to ensure proper contact in the final configuration.

In its initial configuration (Fig.18-a), both faces are piled and the torque spring is stretched on the first axis. It unfolds until it starts to push the tip of the release system, reached in Fig.18-b. As soon as the contact is made, the pin of the first axis retracts, thanks to a tension spring inside the body to the right. A guillotine maintaining the pin in place during the first stage of deployment is disengaged by pushing the tip. After the pin is removed, the magnet brings together both parts of the final joint (Fig.18-c). The joint is now free to rotate perpendicularly to the face plane, as required for the deployment phase (Fig.18-d). 


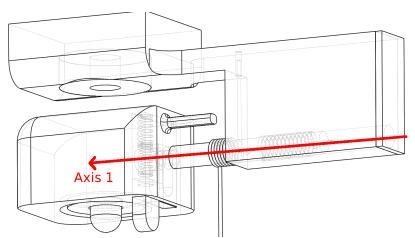

(a)

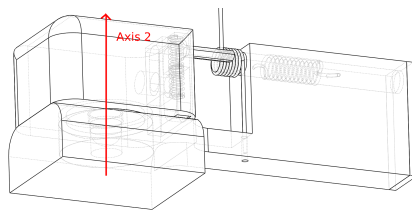

(c)

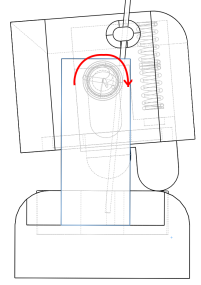

(b)

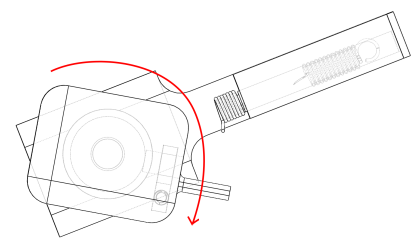

(d)
Fig. 18. Dual phase rotary joint. a- initial state, b- about to release the first axis, $c$ - held together with magnet, $d$ - second rotation axis.

\section{Prototype}

A prototype of the triangular deployable unit of the mechanism proposed in this paper was built using the 3Dprinting technique proposed in [28]. The symmetric extension of each leg of the prototype is shown in Fig.19-a. The design is made to allow quick removal or addition of stages with snap-on axes. Each pulley is glued to the corresponding link and the open belts are installed in the completely extended configuration with a small compressing sandwich part to allow fine (one teeth thick) adjustement. The axes are made of steel for robustness and limited friction and each stage has an upper and lower part thus forming a strong closed profile.

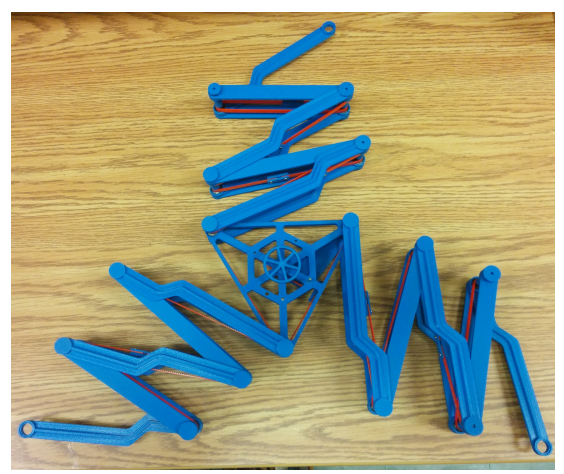

(a)

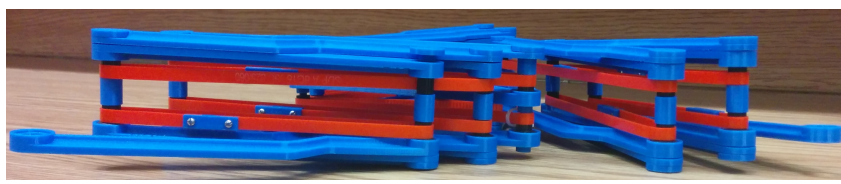

(b)

Fig. 19. Prototype.
The extension concept based on belts and fixed pulleys works correctly. However, the small diameter of the selected pulley allows only 5 teeth to engage at a time and thus making the mechanism prone to slippage. The tension in the belts has to be large enough to compensate for this effect. The plastic parts then bend a little under such tension as shown in Fig.19-b. Manipulation showed good functionality but the actuation from the central position of the triangle is slightly difficult due to the sum of the friction forces in each joint of each leg. Larger pulleys and larger links should be used to overcome the friction and bending problems due to large belt tension.

The prototype also highlights the fact that the actuator has to be selected by taking into account the friction in all joints of the mechanism.

Using revolute joints, clearance may be an issue that should be addressed by simulation and prediction of its impact on the mechanism as discussed in [26,29]. Another important issue is the potential deformation of the links under the compressive loads induced by the belts [30].

\section{Conclusion}

This paper presented the design of $1 \mathrm{DoF}$ expandable mechanisms. First, mechanisms based on rigid links were discussed. It was found that, although such mechanisms can provide large expansion ratios, the latter are limited in practice by the transmission angles. In order to overcome this issue, a new mechanism inspired by the latter but using belts for transmission was introduced. The effect of the different parameters on the expansion ratio of the mechanism was studied and an optimisation problem was formulated. Based on the results, a prototype of a triangular expandable mechanism was built and demonstrated. Finally, a dual-phase joint was introduced in order to further increase the expansion ratio by stacking triangular modules and deploying them sequentially.

For small forces and using appropriate control to compensate for belt elasticity and friction, this mechanism can be very effective and ligthweight. Its performances (robustness and expansion ratio) are expected to be better for larger scale mechanisms.

\section{Acknowledgements}

The authors would like to acknowledge the financial support of the Natural Sciences and Engineering Research Council of Canada (NSERC) and the Canada Research Chair Program.

\section{References}

[1] Ishii, K., 2000. Structural Design of Retractable Roof Structures. WIT Press, Japan.

[2] Gantes, C., 2001. Deployable Structures: Analysis and Design. WIT Press, New-York.

[3] Imbriale, W. A., Gao, S., and Boccia, L., 2012. Space Antenna Handbook. Wiley, USA. 
[4] Pellegrino, S., 2001. Deployable Structures, Vol. 412. Springer-Verlag Wien, New-York.

[5] Hoberman, C., 1991. Radial expansion/retraction truss structures, 06. US Patent 5024031.

[6] Wei, G., and Dai, J. S., 2012. "Synthesis of a family of regular deployable polyhedral mechanisms (dmps)". Latest Advances in Robot Kinematics, pp. 123-130.

[7] Korkmaz, K., 2005. "Generation of a new type of architectural umbrella”. International Journal of Space Structures, 20, pp. 35-41.

[8] Lopatina, A., and Morozov, E., 2009. "Modal analysis of the thin-walled composite spoke of an umbrella-type deployable space antenna". Composite Structures, $\mathbf{8 8}$ i1, pp. 46-55.

[9] Wei, X.-Z., Yao, Y.-A., Tian, Y.-B., and Fang, R., 2006. "A new method of creating expandable structure for spatial objects". Proceedings of Institution of Mechanical Engineers, Part C: Journal of Mechanical Engineering Science, 220, pp. 1813-1818.

[10] Kiper, G., Soylemez, E., and Kisisel, A. O., 2008. "A family of deployable polygons and polyhedra". Mechanism and Machine Theory, 43 i5, pp. 627-640.

[11] Agrawal, S., and Kumar, S., 2002. "Polyhedral single degree-of-freedom expanding structures: design and prototypes". ASME Journal of Mechanical Design, pp. 473-478.

[12] Kovacs, F., Tarnai, T., Fowler, P., and Guest, S., 2004. "A class of expandable polyhedral structures". International Journal of Solids and Structures, 41, pp. 11191137.

[13] Wolhart, K., 1995. "New overconstrained spheroidal linkages.". Proceedings of the 9th World Congress on the Theory of Machines and Mechanism, 1, pp. 149154.

[14] Gosselin, C., and Gagnon-Lachance, D., 2006. "Expandable polyhedral mechanisms based on polygonal one-degree-of-freedom faces". Proceedings of Institution of Mechanical Engineers, Part C: Journal of Mechanical Engineering Science, 220 i7, p. 1011.

[15] Wolhart, K., 2008. "Double-ring polyhedral linkages.". Proceedings of the Conf. on Interdisciplinary Applications of Kinematics, pp. 1-17.

[16] Phillips, J., 1984 \& 1990. Freedom machinery, Vol. 1 \& 2. Cambridge University Press, UK.

[17] Guest, S., and Fowler, P., 2005. "A symmetry-extended mobility rule". Mechanism and Machine Theory, 40 i9, pp. 1002-1014.

[18] Loeb, A., 1991. Space Structures. Birkhauser, Basel, Germany.

[19] Gogu, G., 2005. "Chebychev-grübler-kutzbachs criterion for mobility calculation of multi-loop mechanisms revisited via theory of linear transformations". European Journal of Mechanics A/Solids, 24, pp. 427-441.

[20] Hartenberg, R., and Denavit, J., 1964. Kinematic synthesis of Linkages. McGraw-Hill, New-York.

[21] Ballia, S. S., and Chanda, S., 2002. "Transmission angle in mechanisms (triangle in mech).". Mechanism and Machine Theory, 37.
[22] Tao, D., 1964. Applied Linkage Synthesis. AddisonWesley, MA.

[23] Gosselin, C. M., Sefrioui, J., and Richard, M. J., 1992. "Solutions polynomiales au problème de la cinématique directe des manipulateurs parallèles plans à trois degrés de liberté". Mechanism and Machine Theory, 27 i2, pp. 107-119.

[24] L. Birglen, T. L., and Gosselin, C., 2008. Underactuated robotic hands, Vol. 40. Springer-Verlag Wien.

[25] St-Onge, D., and Gosselin, C., 2014. "Deployable mechanisms for small to medium sized space debris removal". Proceedings of the 24th International Astronautics Congress, Space Debris Symposium, p. 11.

[26] Li, J., Yan, S., Guo, F., and Guo, P., 2013. "Effects of damping, friction, gravity, and flexibility on the dynamic performance of a deployable mechanism with clearance". Journal of Mechanical Engineering Science, 227, pp. 1-13.

[27] Wohlhart, K., 2007. "Cupola linkages". Proceedings of the 12th IFToMM World Congress, pp. 319-324.

[28] Laliberté, T., Gosselin, C., and Côté, G., 2001. "A rapid prototyping framework for fast and cost-effective design of robotic mechanism prototypes". IEEE Robotics and Automation Magazine, 8, pp. 43-52.

[29] Hongwei, G., Jing, Z., Rongqiang, L., and Zongquan, D., 2013. "Effects of joint on dynamics of space deployable structure". Chinese Journal of Mechanical Engineering, 26, p. 861.

[30] Britvec, S., 1995. Stability and Optimization of flexible space structures. Birkhauser, Basel, Germany. 